\title{
Web-based photonics simulator for secondary school students
}

Sam Campbell, Kali Madden, Adam Strickland, Robert Williams, Nemanja Jovanovic, et al.

Sam Campbell, Kali Madden, Adam Strickland, Robert Williams, Nemanja Jovanovic, Benjamin F. Johnston, Judith M. Dawes, "Web-based photonics simulator for secondary school students," Proc. SPIE 9666, 11th Education and Training in Optics and Photonics Conference, 96660V (5 June 2009); doi: $10.1117 / 12.2207975$

SPIE Event: Eleventh International Topical Meeting on Education and Training in Optics and Photonics, 2009, St. Asaph, United Kingdom 


\title{
Web-based photonics simulator for secondary school students
}

\author{
Sam Campbell, Kali Madden, Adam Strickland, Robert Williams, \\ Nemanja Jovanovic, Benjamin F. Johnston and Judith M. Dawes, \\ Centre for Ultrahigh bandwidth Devices for Optical Systems (CUDOS) \\ MQ Photonics Research Centre \\ Physics and Engineering, Macquarie University, NSW 2109, Australia \\ Phone: +61-2-9850-8903, Fax: +61-2-9850-8115, Email: judith.dawes@mq.edu.au
}

\begin{abstract}
In the "real world", Photonics is somewhat invisible to those who rely upon it worldwide. We would like students to connect their everyday experiences of communications with the underlying ideas in Photonics. To do this, we have developed the Photonics Simulator to illustrate to high school students how text or information is coded into binary optical signals which are relayed through photonic communications networks from sender to receiver.
\end{abstract}

Using our simulator, students construct a virtual network, and then test it by sending messages. The messages are coded using ASCII binary code as digital signals in data packets with address headers, which need to be switched, combined, amplified, or delayed to get to their designated address. The students must manage their power budget, correctly target each message address, and avoid collisions of data packets to send their messages uncorrupted and error-free.

We tested an early version of the simulator with five Year 9 and 10 classes. The students provided many constructive comments and their feedback was used to improve the graphical interface of the simulator. We subsequently tested the simulator with 80 Year 9 students in short workshops.

Overall we had a very positive response - it was more fun than a normal class, and interactivity helped students retain information. Students enjoy the visual aspects- they see how messages are delivered, and learn the function of each network component by experiment. Tests of the simulator at the Macquarie Siemens Science Experience were also encouraging, with one student even sneaking back to class to complete his challenges!

\section{INTRODUCTION}

The Centre for Ultrahigh-bandwidth Devices for Optical Systems, CUDOS, is an Australian Research Council Centre of Excellence funded for research on Photonics and Optical Communications, with a further aim to do public communications and outreach, targeting school students, their teachers, industry and others. We aim to raise the profile of Photonics amongst school students in particular, so that they may take their place in our highly connected and communications-rich world. We also hope that engaging school students in this manner will influence them to continue their studies of science.

Our experience in doing outreach is that school students and many of their teachers are quite ignorant of the role that photonics plays in the internet and communications generally; for example, they are surprised to learn that their international data and phone calls are transmitted via light pulses through submarine optical fiber cables. This highlights a need for our outreach activities to be educational as well as promotional, that is, aiming to increase understanding of our research, not just awareness that it exists. 
We also note a number of challenges concerning resourcing (both presenters' time and financial support for their activities) and in assessing the efficacy of our communications activities. These challenges can be addressed by developing shareable media online - such as our Photonics Simulator.

The aim of the simulator is to engage students and to educate them, illustrating for them the principles of coding and transmitting information, explaining the function of each individual component within a communications network, and then allowing the students to work through the simulator challenges to create and test their own photonic network on the computer. Each component is introduced in a graded manner with a challenge to explain its properties, which mimic the properties of real components. A subsidiary aim is to demonstrate to students how science and technology contributes to society, encouraging them to study science and technology in high school and beyond, to continue the work of today's pioneers.

The simulator is based on Flash, which is compatible with both PC and Mac computing platforms. We have included a dictionary of terms to introduce the main components and concepts used in the simulator, and we also included short movies and animations to introduce the game - "Getting started" - and to demonstrate concepts more vividly. We intend that the simulator should become a tool for teachers to use in introducing science students to photonics, and it could also readily be used by the students themselves at home. We also hope that Optics and Photonics researchers around the world could publicize the simulator to their local schools.

In our presentations to school students, we first presented a talk to introduce secondary students to photonic communications systems. We also included other activities such as a worksheet to complete to get the students to work out what each component does before tackling the simulator. In our presentations, we also offered an animation of a photonic chip and a fun freeze-frame movie showing how various photonic components work, and a "real" demonstration of a laser with optical fibre.

In our presentations with the students using the simulator, we deliberately did not direct students as to how to set up their networks as we wanted to give the students an open choice as to how to interact with the simulator. Indeed, we found that most students expected to "hack" rather than be issued with instructions, and many would open the simulator when they were supposed to be busy on another aspect of the presentation. While we have offered the students both the choice of graded challenges or "free play", we have noted that mostly they prefer to use the graded challenges, as they lack the appropriate background to understand the free play option readily.

\section{EFFECTIVE SOFTWARE FOR EDUCATION}

The combination of effective teaching and learning is difficult to achieve. Sometimes educational software addresses perceived teaching needs, rather than increasing student engagement, which can make the integration of such software into the classroom problematic. Effective learning by students requires them to actively participate and be engaged in the process, whereas teaching is often focused on conveying ideas. It is also essential that teachers' needs are considered in order for any software to be adopted by schools. Hence, for educational software to be successfully integrated into the classroom, both the needs of teachers and students must be integrated into the software ${ }^{1}$. Polonoli ${ }^{2}$ suggests that there are seven aspects to creating effective educational software, which are:

- appropriate integration of learning and teaching; .

- contain game-like features

- target to an appropriate grade level

- highly interactive

- friendly interface

- $\quad$ represent the students' world

- not culture-sensitive. 
Of course, educational software must be appropriate for the age, gender and learning style of the students it serves, and not culturally offensive to any group.

Educational software that uses a "game-like" approach has been shown to have a strong cognitive effect on students learning science-based concepts, akin to problem solving in the cognitive processes that are generated ${ }^{3,4}$. According to Randel et al $^{3}$ games foster greater interest from students when compared with traditional forms of instruction. When students are engaged, the game makes class time less boring ${ }^{5}$ and it is more likely they will remember the concepts taught by the game ${ }^{3}$.

On the other hand, other researchers believe that despite games for education being "engaging" for the students, they do not stimulate the constructive reasoning that is required for learning as students are distracted from cognitive reflection on the key concepts. Manske and Conati ${ }^{6}$ suggest that little evidence exists to show that games can trigger learning, unless they are supported by other activities. Disconcertingly, Yeo, et $\mathrm{al}^{7}$ showed that interactive multimedia experiences may not illuminate the key physics concepts for their students, despite interactivity and animated graphics. In their study, "only following researcher intervention, did students develop awareness" of the abstract physics concepts. Hence the level to which the educational software is made "game-like" should be considered carefully, and a total reliance upon the software alone for a student's education is unwise.

\section{DEVELOPMENT OF THE SOFTWARE APPLICATION}

This project started as a jointly-supervised Computing and Physics Masters in Information Technology project in which we chose to develop an educational software package to engage and educate students in Photonics. We developed a simulation program that allowed students to build a simple communication network, and to use concepts from optical networking to send messages through the network. We targeted the game at secondary school students (Years 9-11), to match an appropriate level of the school syllabus. We chose Flash as the delivery platform for the simulator because it can be easily hosted online, it is widely available as a free download, and it offers good graphical rendering without too much computational load. In addition to handling the graphics, Flash provides a scripting interface (ActionScript) and is available to a wide range of machine platforms including both PC and Mac. It offers useful features such as silent exception handling, garbage collection and a rich object model. However, as with many such platforms, one of the biggest problems with Flash is performance - it is limited to small applications with relatively few simultaneous graphics and processes.

The photonics simulator may be viewed at the website:

http://web.science.mq.edu.au/groups/cudos/education/Simulator.html

The Photonics Simulator has been developed to balance the needs of students and teachers. The application does not provide students with specific directional information, rather students are provided with open possibilities which may be guided by a teacher, perhaps via worksheets or modeled answers, or alternatively the students may explore different avenues on their own. The Photonics Simulator has three areas: Watch a video; Build a photonic network; and Photonics dictionary. The videos provide an opportunity to present information on photonic chips in general (the ultimate research goal for CUDOS), or more detailed information on Getting Started in the game. Alternatively the teacher may play a video to the class as a whole. This part of the application does not provide much interactivity, but provides content for the students to prepare them for building their own photonic network.

Building a network gives students a chance to actively learn concepts introduced by their teacher, through class discussions, or by watching the movies above. It provides students with fifteen graded 
challenges to explain and introduce the individual optical components that the students can use in their networks. The challenges start with a simple requirement to connect the source to the receiver, but Challenge 15 combines four sources, four receivers and eleven possible components in a complex networking task. Given a mixed group of abilities, ages and cultures of the target students, we have chosen to include both a graded defined-challenge approach and a free-play interactive approach. Some students work better in a structured environment, while others strongly prefer to test their own ideas without too many constraints. Thus the graded challenges introduce the students to the individual concepts and components of the simulator step by step, whereas the free-play option offers students various components to combine into their own virtual photonics network, which they can test to see if it sends their message correctly.

The free play option offers an open challenge to transfer a message a student has typed, from any of four transmitters to any of four receivers, without the message being scrambled or attenuated too much. To do this the student must connect the sources (laser transmitters) with the various receivers, using photonics components from their "toolkit" and ensure that their messages are always correctly received. We deliberately do not provide instruction on how they should achieve this, forcing the students to think about what they have learned in the graded challenges or in the class lessons, and to test different approaches to the problem. The aim is to frustrate the student slightly, causing "a mild state of cognitive dissonance that will make the content challenging" ${ }^{2}$.

The open-ended free play section also allows the teacher to set extra tasks or challenges for the students to complete, say to build a network that includes a buffer and a switch. The Photonic network builder also achieves the project aim of introducing the students to the basics of communications networks. A student's solution to Challenge 10 is shown in Figure 1 to illustrate some of these ideas. When the students send their messages through their photonics network, the messages are converted to binary code and transmitted through the network as light and dark pulses. Bezier curves allow the photons to travel along the waveguides that were drawn by the student. The light pulses appear gradually fainter as they propagate through more components, and through the waveguides, thus requiring the students to implement amplifiers in their system to manage the power budget. There is a photon speed slider that allows the students to slow down the speed of the photons through the network so they can watch individual pulses flowing through the network, and see them translated back into "real" characters at the receiver. They may notice that sometimes their characters are scrambled because the power has dropped below a threshold of about $70 \%$ and the ASCII code for that character is changed. Each character is represented by an 8-bit code, with a coloured address header to indicate where that character should be sent. This emphasizes the systems aspects of the network design for the students. It is not enough simply to understand the function of a specific component, but the performance of the network as a whole must be assessed.

Finally, the Photonic dictionary allows students to quickly look up the function of a particular component, or clarify a concept such as coding. The definitions enable the interested student to explore in more depth the concepts and ideas provided by the videos and the Build a photonic network section. This dictionary is also a resource to support students completing worksheets or other activities as required by their teachers.

\section{IS THE SIMULATOR EFFECTIVE?}

We performed trials of the Photonics Simulator with 100 Year 9 and 10 students from three schools, to investigate the effectiveness of the Photonics Simulator for educating and engaging the students. Each lesson (50 to 90 minutes) was split into the following components:

- An illustrated talk on photonics including laser and optical fibre demonstration

- A short movie on photonic networks

- Students complete a worksheet using the Photonics dictionary

- Free time to play with Build an optical network 
Before each trial, a pre-survey was conducted by the teacher at the beginning of the lesson, and two questions from this survey were repeated in a post-survey in the last five minutes of the same lesson to test the effectiveness of the lesson. These questions were:

- What is photonics?

- What is the function of an optical amplifier in an optical network?

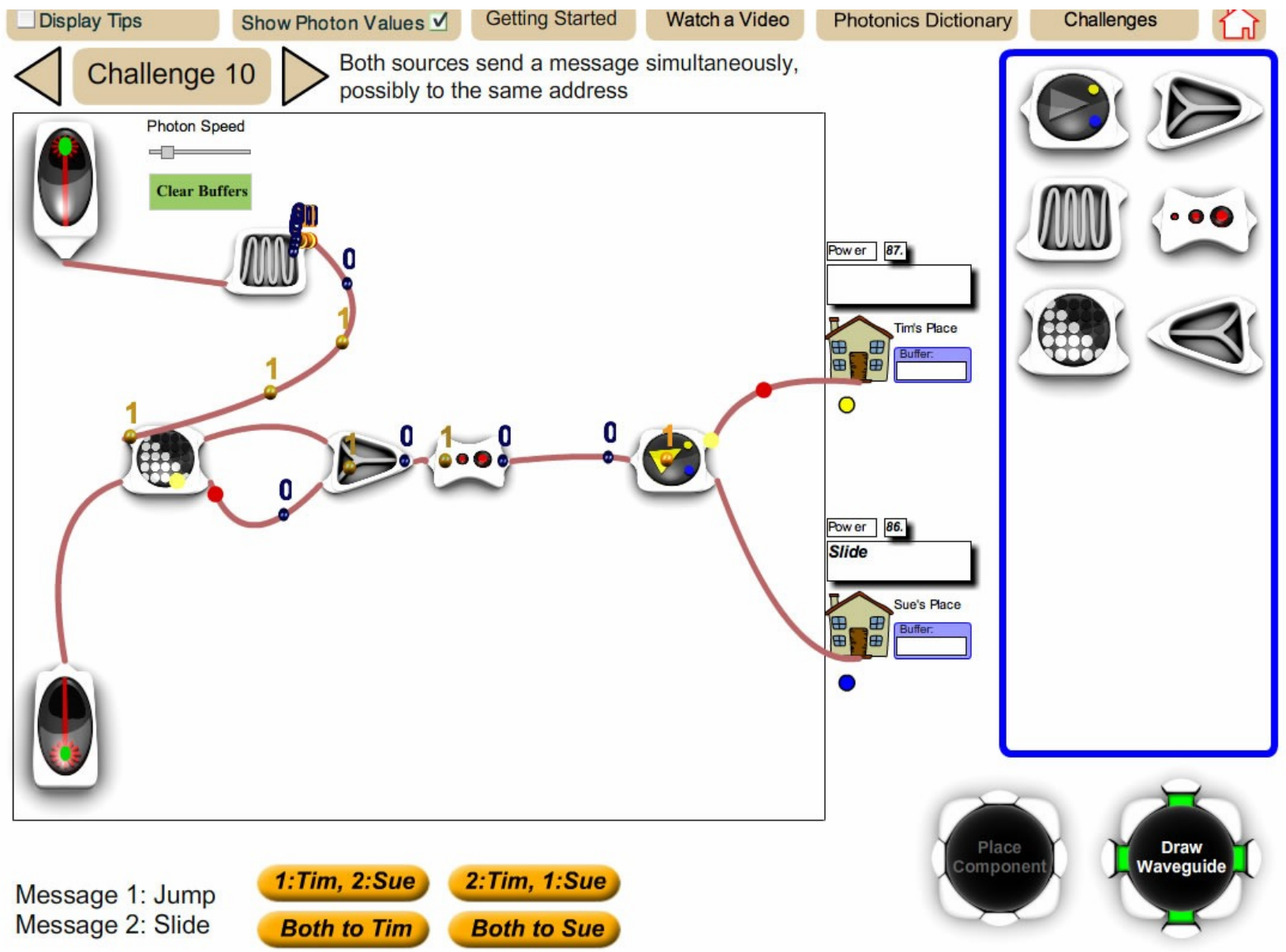

Figure 1: A snapshot from the Building a photonic network section of the application, showing a student's solution to Challenge 10, as a message is being transmitted. Each character is represented by an 8-bit ASCIl code, with a coloured address header, and the ones and zeroes are shown as the light pulses propagate through the network. The student can assess if the network is satisfactory by whether the message is transmitted correctly to the correct receiver.

The change in responses to these two questions is demonstrated by Figures 2 and 3 . There is a significant improvement in the students' responses to both questions after the lesson and experience of the simulator. Responses have been aggregated by gender in this case (girls first two columns, boys second two columns). Examples of "correct" (blue in the Figures) or "half-right" (violet in the Figures) answers for "what is photonics?" are: "science and application of light as a means of transferring information" and "when you turn information into light signals". Examples of "correct" and "half-right" responses to "what is the function of an optical amplifier?" are: "produces an exact copy of a signal with increased intensity" or "to boost the transfer of a signal". In the Figures, pink represents "don't know" and aqua is "wrong". 
As anticipated, when asked if they would enjoy playing scientific computer games in class, almost all students said "yes" (only two boys and two girls said "no"). Rather fewer students would enjoy playing scientific computer games at home: 17 out of 30 girls said "yes", and 29 out of 68 boys said "yes". This may be an important consideration for those of us planning to use computer games for individual outreach to students.

Generally students were very positive about their experience of the computer game. 26 out of 30 female students said they enjoyed the game, and 56 out of 68 male students did likewise. Of the females, 28 out of 30 said they learned something, and 51 out of 68 male students said they learned something.

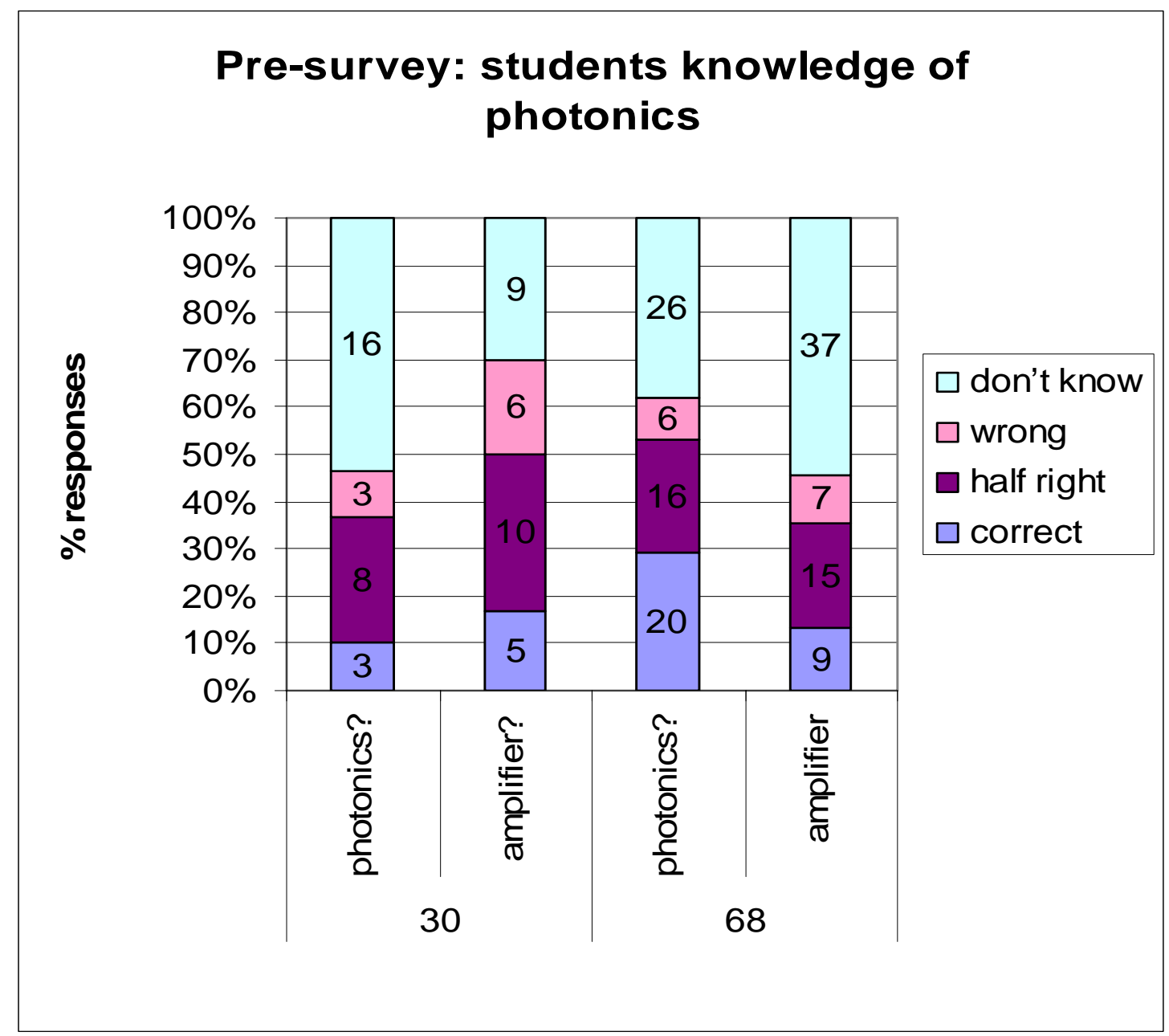

Figure 2: Percentages of students answering Questions 1 and 2 in the pre- survey blue - correct, violet - half right, pink - wrong, aqua - don't know. There were 30 girls and 68 boys surveyed. The questions were: What is photonics? What is the function of an optical amplifier? The overprinted numbers show the actual numbers of responses in each category.

The students were also asked what they liked and didn't like about the Photonics Simulator. The most-liked aspect of the game was the interactive Build a photonic network section, notably the ability to create their own network, and to send messages through it. The fact that it is image-based appeals to many 
students - they can see how messages are delivered, and understand the function of each component in a network by experimenting with it. Dislikes of the game were that it was confusing, needed some more technical or graphical sophistication (e.g. "automate the line drawing" or "use more colour in the dictionary") or too slow. Overall the response was very positive, indicating that it was more fun than a traditional class, and interactivity helped to improve retention of information. It would be interesting to test if the knowledge was retained over subsequent days or weeks! One student correctly commented "since it's a game, you're thinking a lot more, and so grasp the info better". While some wanted more direction, and wanted to be shown a model answer to start, most students enjoyed the openness and self-direction in the application. Another interesting question is whether the graded challenges or the free-play are more effective for promoting students' learning.

Teachers commented that "one of the main things the students learned was the application of science to the real world." "Students enjoyed the interactive nature of the simulation." "They tend not to read instructions, but just want to get into it." "This ... activity can be used to consolidate knowledge of photonics, or as an introduction to expose students to the necessary components." "I tried the simulator and was very impressed. There is so much work into this! I find it very interesting. After seeing the tutorial and trying from the first challenges, I find it easy to use. I think it is the kind of tool that should be used in introduction to photonics classes to provide students with a look of what a network looks like."

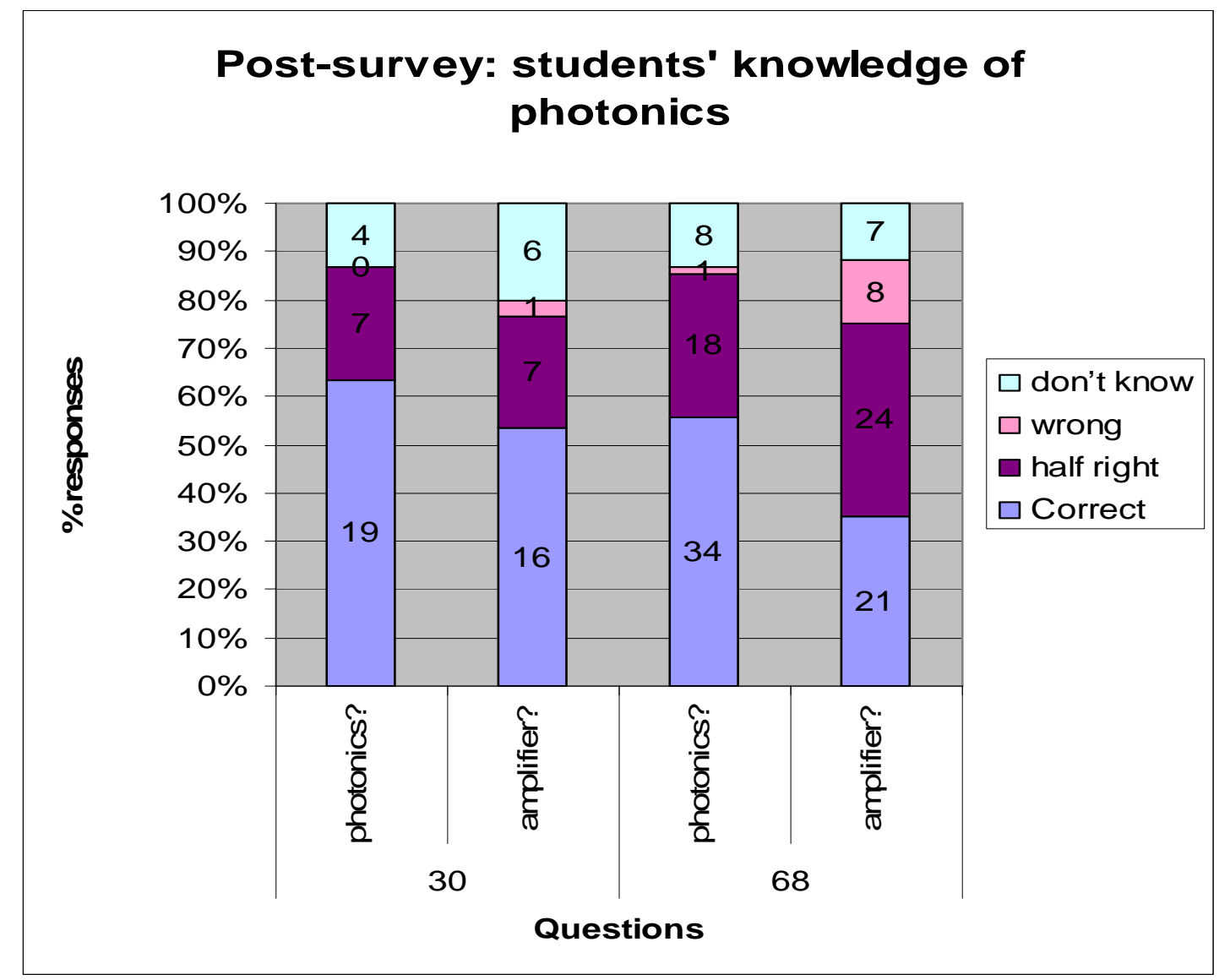

Figure 3: Post-survey responses by students: blue is correct, violet is half-right, pink is wrong and aqua is don't know. The first two columns represent 30 girls, and the second two columns represent 68 boys. The questions were: What is photonics? What is the function of an optical amplifier? 
Approximately 80 students in the 2009 Macquarie Siemens Science Experience also enjoyed playing with the Photonics simulator. These students from Year 9 gain exposure to a range of university science activities during a 3-day series of holiday workshops. They are generally well-motivated to do science, but we found that the majority of the students really enjoyed solving the challenges. (One student even snuck back from another group to finish his challenges!) Some of the student comments that we received on this activity were: "computer activities were really great"; "the program was cool"; "challenging, fun". We did not have the opportunity to assess the students' learning in this activity.

\section{CONCLUSIONS}

The Photonics Simulator was created to engage and educate school students in photonics, with particular focus on incorporating technology into the teaching methodology. We used this application as an aid when teaching the topic, rather than as a stand-alone application. When traditional teaching is combined with additional resources such as a software application, students become actively engaged in the topic through the interactivity of the application, helping to achieve a better understanding of the topic. We are keen to promote the use of the photonics simulator in education and outreach activities, and will continue to trial it in schools. In the future, we would like to make a more overt comparison between electrical and photonic networks, than was possible in this simulator.

\section{ACKNOWLEDGEMENTS}

We are indebted to Carol Oliver, formerly of the Australian Centre for Astrobiology at Macquarie University for her generous sharing of information about how students learn best. Thanks also to the members of CUDOS with whom we had many valuable discussions. We are also grateful to the students and staff of Oakhill College, Arden Secondary School, and MLC School in Sydney and to the students of the Macquarie Siemens Science Experience, for their enthusiastic participation in the trials of the software. This work was completed with the support of the ARC Centres of Excellence Program. We gratefully acknowledge support from the SPIE Education and Outreach Fund.

\section{REFERENCES}

1. Crosier, J.K., Cobb, D. and Wilson, J.R. (2002). Key lessons for the design and integration of virtual environments in secondary science. Computers and Education, 38, 77-94.

2. Polonoli, K.E. (2000). What makes educational software educational? Virginia Society for Technology in Education Journal, 15 44-51.

3. Randel, J., Morris, B., Wetzel, C. \& Whitehill, B. (1992). The effectiveness of games for educational purposes: A review of recent research. Simulations and Games. 23(3). 261-276.

4. Pillay, H., Brownlee, J. and Wilss, L. (1999). Cognition and recreational computer games. Implications for educational technology. J Research on Computing in Education. 32 203-217.

5. Roblyer, M. D., Edwards, J., \& Havrilik, M. A. (1997). Integrating educational technology into teaching. Upper Saddle River, NJ: Prentice-Hall, Inc.

6. Manske M. and Conati C. (2005). Modelling Learning in Educational Games. Proc AIED 05, Proceedings of the 12th International Conference on Al in Education, Amsterdam, July 19-23.

7. Yeo, S., Loss, R., Zadnik, M., Harrison, A. and Treagust, D. (2004). What do students really learn from interactive multimedia? A physics case study. American Journal of Physics, 72, 1351-1358. 